\title{
A Rett syndrome patient with a ring $X$ chromosome: further evidence for skewing of $X$ inactivation and heterogeneity in the aetiology of the disease
}

\author{
Carla Rosenberg ${ }^{*}{ }^{1}$, Cokkie H Wouters ${ }^{2}$, Karoly Szuhai ${ }^{1}$, Rien Dorland ${ }^{3}$, Peter Pearson ${ }^{3}$, \\ Bwee Tien Poll-The ${ }^{4}$, R Max Colombijn ${ }^{5}$, Martijn Breuning ${ }^{6}$ and Dick Lindhout ${ }^{2,3}$ \\ ${ }^{1}$ Laboratory of Cytochemistry and Cytometry, Department of Molecular Cell Biology, Leiden University Medical \\ Center, Leiden, The Netherlands; ${ }^{2}$ Department of Clinical Genetics, Erasmus University Rotterdam, Rotterdam, \\ The Netherlands; ${ }^{3}$ Department of Medical Genetics, University Medical Centre Utrecht, Utrecht, The Netherlands; \\ ${ }^{4}$ Metabolic Department, University Medical Centre Utrecht - Wilhelmina Children's Hospital, Utrecht, The \\ Netherlands; ${ }^{5}$ Department of Paediatrics, Beatrixziekenhuis, Gorinchem, The Netherlands; ${ }^{6}$ Department of Human \\ and Clinical Genetics, Leiden University Medical Center, Leiden, The Netherlands
}

Rett syndrome (RTT) is an X-linked neurodevelopmental disorder, characterised by regression of development in young females. Recently, mutations in the MECP2 gene were found to be present in $80 \%$ of sporadic cases, but in much lower frequency $(<30 \%)$ among familial cases. Several reports claim that the pattern of $\mathrm{X}$ chromosome inactivation $(\mathrm{XCl})$ relates to the penetrance of $\mathrm{RTT}$; in some cases skewed $\mathrm{XCl}$ is seen in Rett patients, and in others it is observed among normal carriers. We present here a case of RTT with a $46, X, r(X)$ in which complete skewed inactivation of the ring was demonstrated. Further, no mutations were found in the MECP2 gene present on the intact $X$. Our data, in conjunction with two previously published cases of $\mathrm{X}$ chromosome abnormalities in RTT, indicate that $\mathrm{X}$ chromosome rearrangements are sporadically associated with RTT in conjunction with extreme skewing of $X$ inactivation. Based on our case and reported data, we discuss the evidence for a second X-linked locus for RTT associated with lower penetrance, and a different pattern of $\mathrm{XCl}$, than for MECP2. This would result in a larger proportion of phenotypically normal carrier women transmitting the mutation for this putative second locus, and account for the minority of sporadic and majority of familial cases that are negative for MECP2 mutations. European Journal of Human Genetics (2001) 9, $171-177$.

Keywords: Rett syndrome; X inactivation; Ring X

Introduction

Rett syndrome (RTT) is a progressive neurodegenerative disorder affecting 1/10 000-1/15 000 females. Diagnosis is based on clinical signs, including mental retardation, autistic behaviour, epileptic seizures, loss of purposeful hand use and deceleration of head growth. ${ }^{1,2}$ Although most cases are

*Correspondence: Carla Rosenberg, Laboratory of Cytochemistry and Cytometry, Department of Molecular Cell Biology, Leiden University, Wassenaarseweg 722333 AL Leiden, The Netherlands.

Tel: +31 715276190; Fax: +31 71 5276180; E-mail: c.rosenberg@lumc.nl Received 15 November 1999; revised 6 November 2000; accepted 14 November 2000 sporadic, in the limited familial cases reported inheritance is always through the maternal line. ${ }^{3-6}$ Linkage studies pointed to $\mathrm{Xq} 28$ as the most likely region to harbour a RTT gene. . $^{3,4,8}$ Using a systematic gene screening approach in the area, it was recently found ${ }^{9}$ that about $80 \%$ of the sporadic RTT patients present mutations in the X-linked MECP2 gene. ${ }^{10-13}$ The majority of these cases are de novo. ${ }^{12,14}$ Although familial cases are rare, and the data are still limited, it is becoming evident that only a small proportion of familial cases $(<30 \%)$ present mutations in the MECP2 gene. ${ }^{12,15,16}$ Heterogeneity of the syndrome was suggested, and a second RTT locus was hypothesised to be associated to the MECP2 negative patients; ${ }^{10}$ the authors suggest that the frequency of some 
RTT symptoms, such as epilepsy, might differ between groups with and without a mutated MECP2 allele, although differences were not found to be statistically significant.

$\mathrm{X}$ chromosome inactivation (XCI) seems to play an as yet, unknown role in the aetiology of RTT. XCI was found to be skewed in RTT patients as a group, albeit not in every patient, and the skew always favoured inactivation of the paternal. ${ }^{17-19}$ As a complicating matter, skewing of $\mathrm{X}$ inactivation was sometimes reported in normal carriers instead of in the probands. After screening for MECP2 mutations became available, a new pattern seemed to emerge: RTT patients with MECP2 mutations, which are the majority of the sporadic and minority of the familial RTT cases, present random XCI, while the mildly symptomatic or asymptomatic females carrying the MECP2 mutations were reported to present skewing of $X$ inactivation. ${ }^{14,16,20}$ Skewing of XCI, however, is also found in RTT patients ${ }^{15}$ who do not appear to carry MECP2 mutations.

XCI skewing might occur by chance, because of variations in the normal distribution of $\mathrm{X}$ inactivation in the female population. Alternatively, it might be associated with polymorphisms of the XIST (X inactivation specific transcript) locus affecting the probability of a given $\mathrm{X}$ to inactivate. $^{21,22}$ Skewing of XCI is also seen when the X chromosome is structurally rearranged; as a rule, the pattern that leads to the most balanced expression of the genome is selected. Two cases of RTT with balanced X/autosome translocations were previously reported, both with breakpoints in the short arm of the $X$ chromosome. ${ }^{23,24}$ The breakpoints differed in the two cases (Xp11.2 and Xp21.3).

We describe here a case of a RTT patient with $46, \mathrm{X}, \mathrm{r}(\mathrm{X})$ karyotype, and with breakpoints different to the two other reported RTT cases with X chromosome rearrangements. We investigated the presence and expression of XIST in the $r(X)$, and screened the cells of the patient for MECP2 mutations. The data from our patient is consistent with a proposed heterogeneity in the aetiology of RTT.

Based on our results and data from the literature, we present a model that takes into account the differences in frequency of $M E C P 2$ mutations in familial and sporadic cases, and the respective patterns of XCI. The model involves two separate X-linked RTT loci: one with mutations in MECP2 giving rise to a highly penetrant and predominantly sporadic RTT phenotype; and another, as yet unidentified locus, in which mutations give rise to a low penetrant phenotype with many mildly or unaffected carriers and exhibiting a much higher proportion of familial cases.

\section{Patient and methods \\ Patient}

The propositus is the second child of healthy nonconsanguineous parents. Her sister and half-brother are normal. The patient was born after an uneventful pregnancy, with a weight of $3500 \mathrm{~g}$ and length of $50 \mathrm{~cm}$. During the first 3 months of postnatal life, development and behaviour were reportedly normal. At the age of 3 months, shortly after feedings at night had been skipped, convulsions occurred, and were found to be associated with periods of hypoglycaemia without hyperinsulinaemia. Extensive screening for inborn errors of metabolism and endocrine disturbances did not reveal any consistent abnormality.

The subsequent course was characterised by psychomotor delay and severe mental retardation, the development of epilepsy, and failure to thrive, which eventually necessitated treatment with feeding through a percutaneous gastrostomy. Growth was delayed, and her height, which was normal at birth, was at or below the 5 th centile from 2 years onwards.

Neurological examination at the age of 3 years showed diffuse hypotonia with slight hypertonia of the lower extremities, and stereotype movements of the hands. At this age, additional metabolic examinations were performed, and notably revealed a disturbed lactate/pyruvate ratio, combined with a normal glucose/insulin ratio, following an oral glucose tolerance test $(2 \mathrm{~g} / \mathrm{kg})$, indicating a possible defect of the respiratory chain without paradoxical ketonaemia.

At the age of 5 years and 8 months, the patient and her mother were referred for syndrome evaluation and genetic counselling, in which microcephaly was disclosed $(-2 \mathrm{SD}$, as compared to average head circumference during first year of life). The hands showed stereotypic movement, together with stereotypic turning around of the head. Both the history and examination gave evidence for persistent automutilative behaviour. In general, the behaviour impressed as 'autistiform'.

On the basis of psychomotor regression after a period of normal development, severe mental retardation, growth deceleration, loss of purposeful hand skills with appearance of stereotypical hand movements, epilepsy and microcephaly, $^{25}$ the diagnosis of RTT was made.

\section{Classical and molecular cytogenetics}

Metaphases were obtained from both peripheral blood lymphocytes and skin fibroblasts, by standard procedures. FISH studies with probes for centromeric sequences of the $\mathrm{X}$ $\left(\mathrm{pBAM} 45^{26}\right.$ ) and Y (pDP97 ${ }^{27}$ ) chromosomes were performed according to established procedures. ${ }^{28,29}$ Hybridisation for telomere sequences was performed with a PNA probe $\left(\left(\mathrm{C}_{3} \mathrm{TA}_{2}\right)_{3}\right.$ - a kind gift from DAKO, Glostrup, Denmark), following the procedures described previously. ${ }^{30}$

Probes for both the short and long arm of the $\mathrm{X}$ chromosome were used to FISH-map the breakpoints in the rearranged chromosome. The PAC probe 92E23, containing the full XIST (X inactivation Specific Transcript) DNA sequence was used to investigate the presence of XIST sequences by FISH. The probes used were obtained either from the CEPH library, ${ }^{31}$ from the Washington University library $^{32}$ or from Roswell Park Cancer Institute (RPCI-1 and RPCI-6 Human PAC Libraries). The DNA for the probes was 
kindly provided by the Genome Technology Centre (Department of Human and Clinical Genetics, Leiden University Medical Center, The Netherlands). Table 1 shows a list of the probes used and their map location on the $\mathrm{X}$ chromosome.

\section{In situ RNA XIST studies}

For in situ detection of XIST RNA, skin fibroblasts of the patient were grown on slides and fixed for RNA studies. The probe PXIST-C6, containing the full cDNA sequence of XIST, $^{33}$ was used to detect XIST RNA in the interphase fibroblasts. The probe was labelled with Digoxigenin-11dUTP (Boehringer Mannheim, Mannheim, Germany) by standard nick translation, and detected with mouse antidigoxigenin followed by rabbit anti-mouse-FITC (Sigma, St Louis, MO, USA). The procedure was performed as described previously. ${ }^{34}$ YAC 917e6 and YAC 681F6 (see Table 1) were labelled with Biotin-11-dUTP (Sigma, St Louis, MO, USA) and detected with streptavidin-Cy3 (Vector, Burlingame, CA, USA). Two double-hybridisations were performed: one with PXIST-C6 and YAC917e6, and another with PXIST-C6 and YAC681F6.

\section{Determination of parental origin of the ring $X$ by methylation studies}

We investigated the methylation status of the highly polymorphic CAG-repeat region in the androgen receptor gene to determine the parental origin of the ring. The procedure was performed as described previously. ${ }^{35}$ Briefly, PCR products from the androgen receptor polymorphic region were generated from DNA samples from the mother and the child (the father was unavailable), and their length determined with the ALF polyacrylamide gel electrophoresis system (Amersham-Pharmacia). DNA from the child was then digested with the methylation-sensitive enzyme CFO1, which only cleaves unmethylated DNA. A CFO1 site is located within the DNA sequence amplified by the primers, and is methylated in the inactive $\mathrm{X}$ and unmethylated in the active X. Following enzyme digestion, only the methylated DNA is intact for PCR amplification.

\section{Screening for mutations in the gene encoding} methyl-CpG-binding protein 2 (MeCP2)

Point mutation analysis was kindly provided by the laboratory of Prof Huda Zoghbi (Howard Hughes Medical Institute, Baylor College of Medicine), as described previously. 9,16,20 The coding region of the MECP2 gene was sequenced in both orientations.

\section{Results}

Classical and molecular karyotype

Classical banding cytogenetic analysis revealed a 46,X,+mar karyotype in the 82 metaphases analysed (32 in lymphocytes and 50 in fibroblasts), that was possibly a remnant of the other sex chromosome.

Hybridisation with the Y-centromere probe showed no hybridisation signal, while hybridisation with a probe for the $\mathrm{X}$-centromere showed fluorescent signals both on the normal $\mathrm{X}$ and on the marker (100 metaphases from fibroblasts, all of them containing the ring chromosome). The $\mathrm{X}$-derived marker was about $1 / 10$ the size of a normal $X$ and the morphology suggested it to be a small ring (Figure 1a). In addition, the marker chromosome showed no detectable telomeric sequences (Figure $1 \mathrm{~b}$ ), confirming that the marker was a ring chromosome.

All probes used to map the breakpoints on the ring chromosome showed hybridisation in the expected location on the normal X chromosome. Table 1 shows the presence or absence of hybridisation signal on the ring $X$ for the probes used. None of the short arm probes used showed hybridisation signal on the ring (Figure 2), indicating that the breakpoint in the short arm is proximal to Xp11.3. For the long arm of the ring chromosome, the breakpoint was found to be at or distal to Xq13. A probe at Xq26 was used to confirm that the breakpoint was proximal to MeCP2 gene

Table 1 Summary of the FISH probes used, their location and their presence $(+)$ or absence $(-)$ on the ring $X$

\begin{tabular}{|c|c|c|c|}
\hline Probes & Location & Source & FISH signal on the ring $X$ \\
\hline PNA $\left(C_{3} \mathrm{TA}_{2}\right)_{3}$ repeat & telomeres & DAKO, Glostrup (Denmark) & - \\
\hline YAC 951D11 & Xp22 & CEPH library & - \\
\hline YAC 681F6 & $\mathrm{Xp} 22.1$ & CEPH library & - \\
\hline YAC 912G5 & Xp21.3 & CEPH library & - \\
\hline A295G1 & $\mathrm{Xp} 21.3$ & Washington library & - \\
\hline PAC 262D19 I & $\begin{array}{l}\text { exon } 3-7 / \text { DMD locus } \\
\text { Xp21.2 }\end{array}$ & RPCI-6 library & - \\
\hline PAC 95C20 & Xp11.3-11.4 & RPCI-1 library & - \\
\hline PAC $230 G 1$ & Xp11.3 & RPCI-1 library & - \\
\hline pBAM 45 & $\mathrm{X}$ centromere & & + \\
\hline YAC 92E23 & Xq13.2/XIST locus & RPCI-1 library & + \\
\hline YAC 917E6 & $\mathrm{Xq} 1.3$ & CEPH library & + \\
\hline PAC196E23 & $X q 26.1-X q 27.2$ & RPCI-1 library & - \\
\hline
\end{tabular}

Electronic database information - The Sanger Centre: http://www.sanger.ac.uk/DataSearch.

RPCI Human PAC Library: http://bacpac.med.buffalo.edu/humalmaleall.htm 
(Xq28). The absence of a signal on the marker demonstrates, under the assumption that we are dealing with a simple 2break rearrangement, that this distal region of the long arm is deleted.
Hybridisations with the XIST (X inactivation Specific Transcript) genomic probe showed signals both on the normal X and ring chromosomes (Figure 3), and demonstrate the presence of XIST sequences on the ring X.
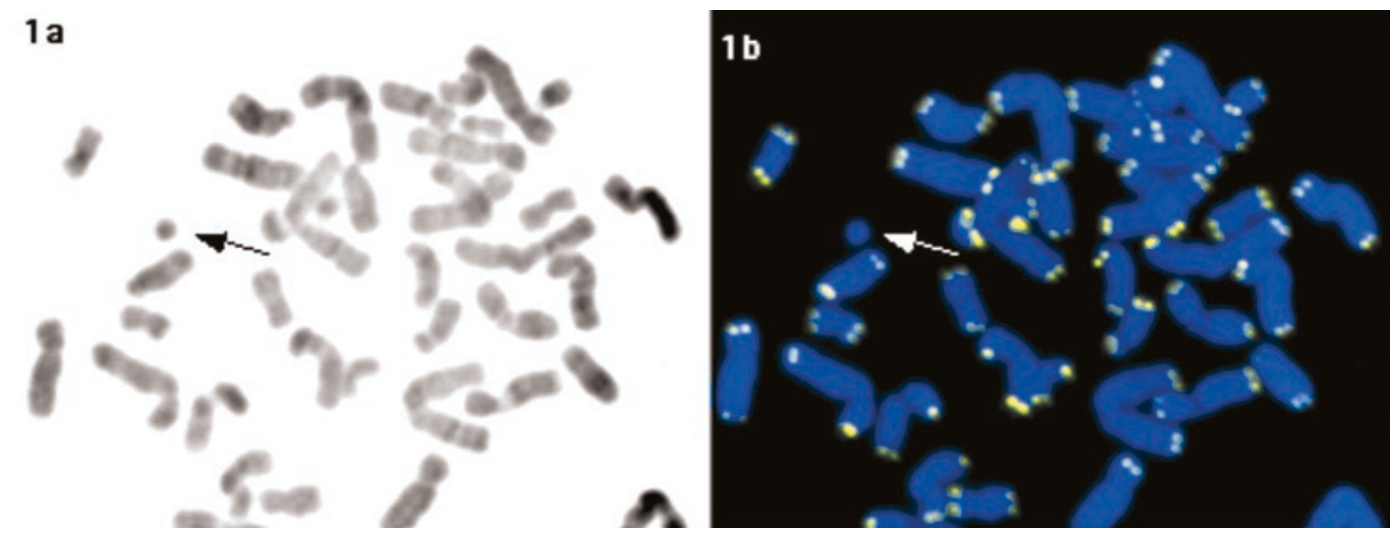

Figure 1 Reverse dapi-banding (a) and fluorescence image (b) of metaphases of the Rett syndrome patient hybridised with telomerespecific probe. The ring (arrow) presents no telomere signals.
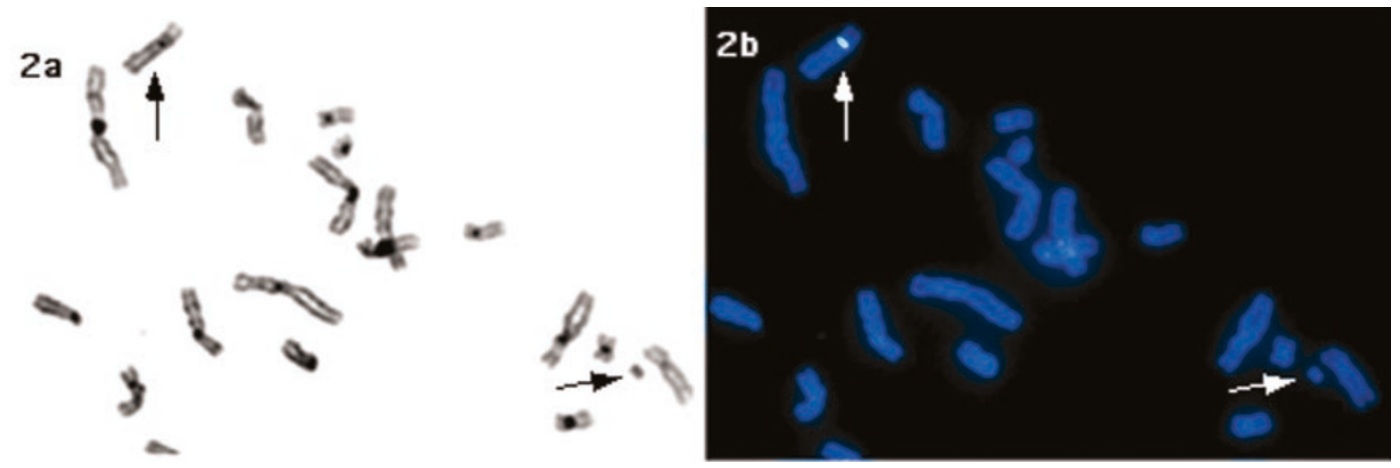

Figure 2 Reverse dapi-banding (a) and fluorescence image (b) of metaphases of the Rett syndrome patient hybridised with a probe (A295G1) in p21.3. A signal can be seen on normal $X$, but it is absent on the ring $X$ (arrows).
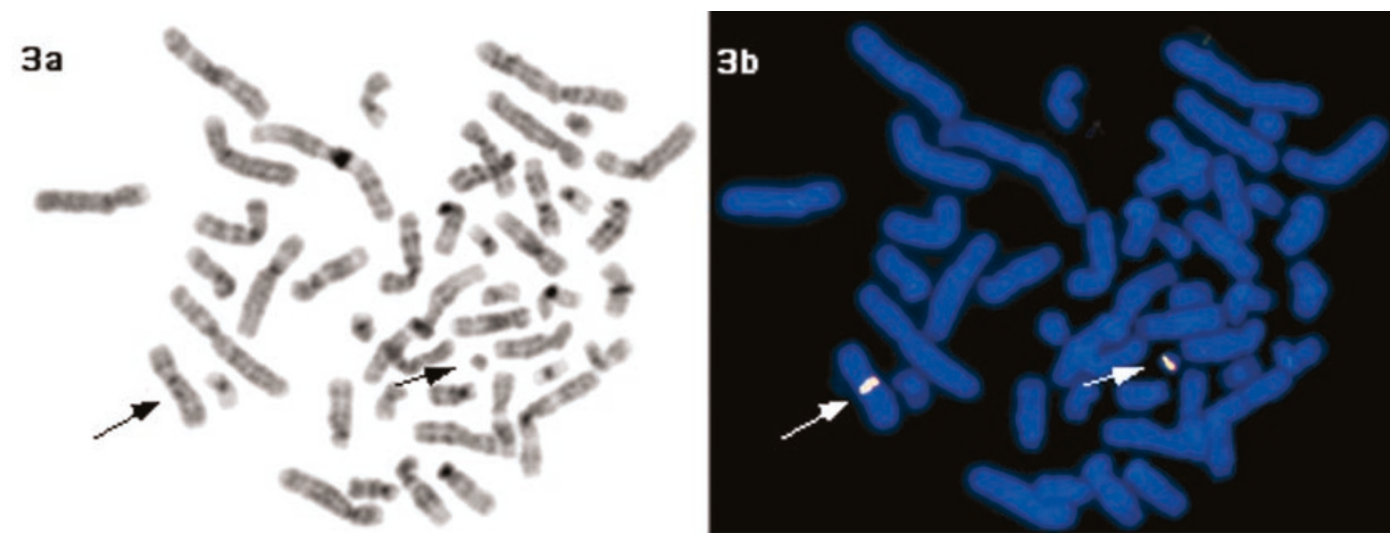

Figure 3 Reverse dapi-banding (a) and fluorescence image (b) of metaphases of the Rett syndrome patient hybridised with a probe for XIST. Signals can be seen both on the normal X and on the ring (arrows). 


\section{In situ RNA XIST studies}

In order to assess the $\mathrm{X}$ chromosome inactivation pattern in this patient, we investigated XIST transcription by in situ detection of XIST RNA, which coats the inactive $X .{ }^{36}$ The simultaneous detection of YAC 917e6 (red signal), which is present on both homologues (see Table 1), and XIST RNA (green signal), shows that one of the $\mathrm{X}$ chromosomes colocalised with the RNA signal (Figure 4a). The simultaneous detection of a sequence only present on the normal X (YAC 681F6 -red) and XIST RNA (green signal) shows that the signals do not co-localise, demonstrating that the normal $\mathrm{X}$ was active (Figure 4b) and the ring inactive in all 20 cells examined.

\section{Determination of parental origin of the ring $\mathrm{X}$ by} methylation studies

Length evaluation of the PCR products from the androgen receptor gene showed that the mother was homozygote for the CAG-repeat polymorphism and the child heterozygote. After digestion of the patient DNA with the methylationsensitive enzyme, just the allele from the child that differs from her mother's remained, indicating that the nonmaternal allele is always methylated, ie, inactive. We concluded that the ring $\mathrm{X}$, which is always inactive, is of paternal origin.

\section{Screening for mutations in the gene encoding} methyl-CpG-binding protein 2 (MeCP2)

No mutation was found after the whole coding region of the MECP2 gene was sequenced in both orientations.

\section{Discussion}

We present a Rett syndrome patient with a 46,X,+mar karyotype. The marker was found by FISH to be an X-derived ring lacking detectable telomeric sequences. From the two previous cases of RTT associated with X rearrangements, ${ }^{23,24}$ only the one with the Xp21.3 breakpoint ${ }^{24,37}$ was studied at the molecular level. Both the YAC spanning the Xp21.3 breakpoint (YAC A295G1) and a 13 Mb more proximal (The Sanger Center) probe on the short arm (YAC 230G1), were deleted in our ring, and demonstrated that the breakpoint in the short arm of the ring lies significantly proximal to that reported by Zoghbi et al. ${ }^{24}$

Deleted X chromosomes are almost always preferentially inactivated. Exceptions are ring X chromosomes that cannot undergo inactivation, either because the XIST gene or other sequences on the $\mathrm{X}$ chromosome required for its expression were affected by the rearrangement. ${ }^{38,39}$ We examined the presence of XIST DNA in this patient, and found the ring to contain XIST sequences. Furthermore, the ring was found to express XIST in all cells examined, ie, to be inactived. Other than symptoms of Turner's syndrome, no other major malformations are generally associated with inactive ring $X$ 's. In this case, given the large size of the deletions on the long and short arms of the X, Turner syndrome symptoms might be expected. Although the patient presented growth retardation after 2 years, her height was normal at birth, and this combined with a subsequent deceleration of growth is more compatible with a Rett than a Turner phenotype. As the patient has not yet reached puberty, sexual development cannot be evaluated. Although the cytogenetic studies demonstrated the ring to be present in all lymphocytes and skin fibroblasts examined, the presence of a normal 46, XX cell population in other tissues cannot be excluded.

Sequencing of the entire coding regions of MECP2 failed to reveal mutations. Mutations in the coding regions of MECP2 are not found in at least $20 \%$ of the sporadic and in the majority of the familial cases. ${ }^{10-12,15,16}$ Bienvenu et al. ${ }^{10}$ suggested the possible involvement of a second X-linked gene in RTT, and considered that the frequency of RTT features might differ among patients with and without MECP2 mutations.

Based on literature information and data from our own patient, we propose a model invoking a second X-linked gene for Rett syndrome and an association of differential XCI in relation to disease phenotype expression. The following data support our model:
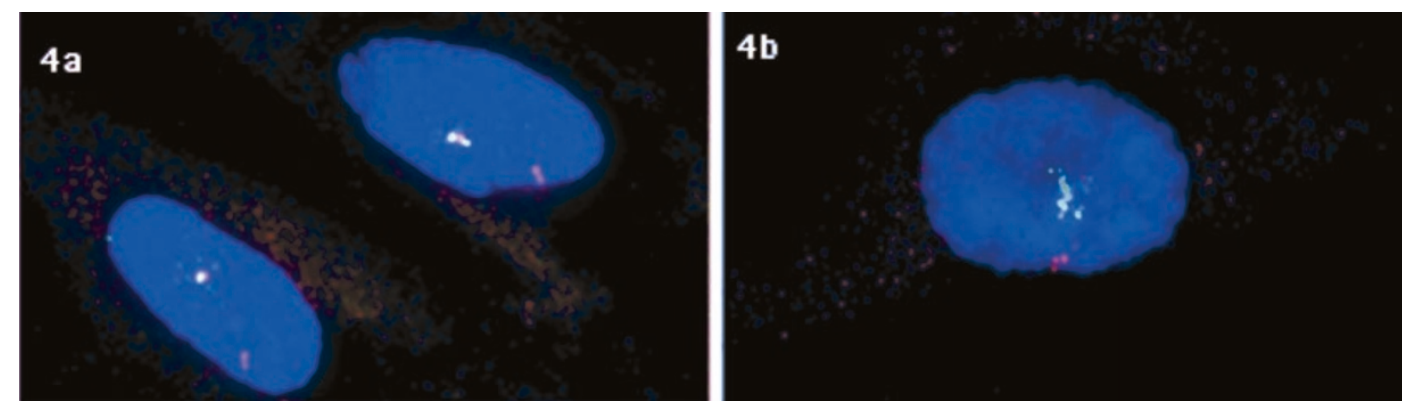

Figure 4 Interphase preparations of the patient. (a) YAC 917 e6 (red signals), present on the ring and normal homologue, and XIST RNA (green signal). The RNA signal co-localises with one of the X chromosomes. (b) YAC681F6 (red signal), which is present on the normal $X$ but deleted on the ring $X$, and XIST RNA (green signal). The signals do not co-localise, indicating that the normal $X$ is active and the ring inactive. 
(1) The frequency of $M E C P 2$ mutations clearly differs between familial and sporadic cases with a low frequency in familial $(<30 \%)$ and a high frequency in sporadic cases $(\sim 80 \%){ }^{12,14-16}$

(2) The limited data available on the relationship of XCI to MECP2 mutations suggest that Rett patients and asymptomatic carriers present different patterns of XCI dependent upon whether or not they are proven carriers of MECP2 mutations and whether they manifest the Rett phenotype:

(a) The majority of MECP2 mutations are de novo. ${ }^{12,14}$ Most MECP2 mutation carriers exhibit the Rett phenotype implying that MECP2 mutations are normally highly penetrant. They exhibit random $\mathrm{XCI}$, and seldom transmit the mutation to the next generation. Rare cases of asymptomatic or mildly symptomatic MECP2 mutation carriers frequently present skewed XCI, ${ }^{14,16,20}$ suggesting that the high penetrance of MECP2 mutations has been ameliorated by preferential inactivation of the MECP2 mutated $X$.

(b) A second disease locus with a lower penetrance and expressivity for the Rett phenotype than MECP2 could account for the MECP2 mutation negative cases and the occurrence of familial transmission in a much larger group of families than those with MECP2 mutations. This putative second locus should exhibit a higher frequency in asymptomatic carriers than MECP2 mutations. The model predicts that asymptomatic carriers for mutations at the second locus will generally exhibit random XCI and those with a Rett phenotype, a skewed XCI. This prediction is supported by the report that MECP2 negative patients frequently exhibit a skewed XCI. ${ }^{15}$

(c) When expressed in all cells, MECP2 mutations do not seem to result in the Rett phenotype: in males, MECP2 mutations result in the severe non-Rett phenotype of encephalopathy. ${ }^{14}$ Further, a severe under-representation of males in both MECP2 mutation positive and negative families indicates a severely reduced viability.

Our patient presents the Rett phenotype, an absence of MECP2 mutation, and a completely skewed XCI, indicating that a different mechanism than MECP2 mutation is causal to her Rett phenotype. The presence of a normal 46, XX cell population in tissues other than those examined of our patient cannot be excluded and such tissues could, theoretically, carry a MECP2 mutation. However, an X chromosome rearrangement associated with extreme skewing of XCI in a patient presenting a X-linked disease, in combination with absence of a MECP2 mutation or mosaicism in the tissues investigated, is unlikely to be coincidental.

Wan et al. ${ }^{14}$ pointed out that the families that were originally used for the linkage analyses leading to the identification of a locus in Xq28, were subsequently shown to carry mutations for $M E C P 2$, implying that the putative second locus could be located anywhere on the $\mathrm{X}$ chromosome.

The patient presented here clearly belongs to the second category, in which a classical RTT phenotype is present with no detected MECP2 mutations, and a completely skewed XCI. Given our model, cases of $\mathrm{X}$ chromosome rearrangements that are sporadically associated with the RTT phenotype are explicable by the extreme skewing of $\mathrm{X}$ inactivation induced by the chromosome rearrangement in association with mutations in an additional RTT X-linked gene with the disease allele carried on the normal, active $\mathrm{X}$. This is a different mechanism to that commonly encountered for $\mathrm{X}$ linked disorders associated with $\mathrm{X}$ chromosome rearrangements where abnormal gene expression is produced by breakpoint disruption or partial functional disomy of the $\mathrm{X}$.

Females with rearranged X chromosomes and RTT have the RTT allele expressed virtually in every cell, similarly to hemizygotos. A question not explained by this model is why males carrying a RTT allele for this second putative gene and presenting a phenotype similar to RTT have not been described in MECP2 mutation negative families. There are two possible explanations for this: either that expression of both $\mathrm{X}$ homologues in the period prior to $X$ inactivation or expression of genes on an otherwise inactivated $\mathrm{X}$, is necessary for survival in the presence of this RTT allele. This latter explanation is intriguing because it implies that such 'survival' genes must be confined to the pericentromeric $\mathrm{X}$-region retained in the small ring $X$ chromosome described here.

This case supports the existence of two different loci in Rett syndrome, and in which differential patterns of XCI contribute to defining the Rett phenotype. For MECP2 mutations, expression in random XCI levels is enough to present a full phenotype, while not fully symptomatic females will exhibit a skewed XCI pattern with preferential inactivation of the chromosome carrying the RTT allele. The majority of cases are new mutations, and normal carriers are rare. A second putative RTT gene can account for the minority of sporadic and majority of familial cases that are negative for MECP2 mutations. To manifest as RTT, the RTT allele should be expressed in a majority of cells, ie, be associated with skewing of XCI involving preferential inactivation of the normal X. Most carriers of this mutation would, therefore, be asymptomatic. Refinement of X-linkage analyses in the families that are negative for MECP2 mutations should shed further light on the location of this second locus.

\section{Acknowledgements}

We thank Johan den Dunnen and Marlies van den Berg for providing the DNA for the FISH experiments, Leendert Looijenga for the cDNA XIST probe and Elmar de Pauw for the PNA hybridisation. We thank Prof. Huda Zoghbi for providing the mutation analyses of MECP2 gene. We also thank Eric Geelen for technical assistance. 


\section{References}

1 Armstrong DD: Review of Rett syndrome. J Neuropathol Exp Neurol 1997; 56: $843-849$.

2 Clarke A: Rett syndrome. J Med Genet 1996; 33: 693-699.

3 Sirianni N, Naidu S, Pereira J, Fernando R, Hoffman EP: Rett syndrome: Confirmation of X-linked dominant inheritance, and localization of the gene to Xq28. Am J Hum Genet 1998; 63 $1552-1558$.

4 Xiang F, Zhang Z, Clarke A et al: Chromosome mapping of Rett syndrome: a likely candidate region on the telomere of Xq. Med Genet 1998; 35: 297-300.

5 Ellison KA, Fill CP, Terwilliger $\mathrm{J}$ et al: Examination of $\mathrm{X}$ chromosome markers in Rett syndrome: exclusion mapping with a novel variation on multilocus linkage analysis. Am J Hum Genet 1992; 50: 278 -287.

6 Schanen NC, Dahle EJ, Capozzoli F, Holm VA, Zoghbi HY, Francke U: A new Rett syndrome family consistent with $\mathrm{X}$ linked inheritance expands the $\mathrm{X}$ chromosome exclusion map. Am J Hum Genet 1997; 61: 634-641.

7 Webb T, Clarke A, Hanefeld F, Pereira JL, Rosenbloom L, Woods CG: Linkage analysis in Rett syndrome families suggests that there may be a critical region at Xq28. J Med Genet 1998; 35: $997-1003$.

8 Wan M, Francke U: Evaluation of two X chromosomal candidate genes for Rett syndrome: glutamate dehydrogenase-2 (GLUD2) and rab GDP-dissociation inhibitor (GDI1). Am J Med Genet 1998; 78: $169-172$.

9 Amir RE, Van dV, I, Wan M, Tran CQ, Francke U, Zoghbi HY: Rett syndrome is caused by mutations in X-linked MECP2, encoding methyl- CpG-binding protein 2 [see comments]. Nat Genet 1999; 23: $185-188$

10 Bienvenu T, Carrie A, de Roux N et al: MECP2 mutations account for most cases of typical forms of Rett syndrome2. Hum Mol Genet 2000; 9: 1377-1384.

11 Huppke P, Laccone F, Kramer N, Engel W, Hanefeld F: Rett syndrome: analysis of $M E C P 2$ and clinical characterization of 31 patients. Hum Mol Genet 2000; 9: 1369-1375.

12 Cheadle JP, Gill H, Fleming N et al: Long-read sequence analysis of the MECP2 gene in Rett syndrome patients: correlation of disease severity with mutation type and location. Hum Mol Genet 2000; 9: 1119-1129.

13 De Bona C, Zappella M, Hayek G et al: Preserved speech variant is allelic of classic Rett syndrome [In Process Citation]. Eur J Hum Genet 2000; 8: 325 - 330 .

14 Wan M, Lee SS, Zhang X et al: Rett syndrome and beyond: recurrent spontaneous and familial MECP2 mutations at CpG hotspots. Am J Hum Genet 1999; 65: 1520-1529.

15 Xiang F, Buervenich S, Nicolao P, Bailey ME, Zhang Z, Anvret M: Mutation screening in Rett syndrome patients. I Med Genet 2000; 37: 250-255.

16 Amir RE, Van dV, I, Schultz R et al: Influence of mutation type and $\mathrm{X}$ chromosome inactivation on Rett syndrome phenotypes [In Process Citation]. Ann Neurol 2000; 47: 670-679.

17 Krepischi AC, Kok F, Otto PG: X chromosome-inactivation patterns in patients with Rett syndrome. Hum Genet 1998; 102 319-321.

18 Camus P, Abbadi N, Perrier MC, Chery M, Gilgenkrantz S: X chromosome inactivation in 30 girls with Rett syndrome: analysis using the probe. Hum Genet 1996; 97: 247-250.

19 Camus P, Abbadi N, Gilgenkrantz S: X inactivation in Rett syndrome: a preliminary study showing partial preferential inactivation of paternal X with the M27 beta probe [letter]. Am J Med Genet 1994; 50: 307-308.

20 Van dV, I, Zoghbi HY: Methyl-CpG-binding protein 2 mutations in Rett syndrome. Curr Opin Genet Dev 2000; 10: 275 - 279.

21 Plenge RM, Hendrich BD, Schwartz C et al: A promoter mutation in the XIST gene in two unrelated families with skewed Xchromosome inactivation. Nat Genet 1997; 17: 353-356.
22 Naumova AK, Plenge RM, Bird LM et al: Heritability of X chromosome-inactivation phenotype in a large family [see comments]. Am J Hum Genet 1996; 58: 1111-1119.

23 Journel H, Melki J, Turleau C, Munnich A, de Grouchy J: Rett phenotype with $\mathrm{X} /$ autosome translocation: possible mapping to the short arm of chromosome X. Am J Med Genet 1990; 35: $142-147$.

24 Zoghbi HY, Ledbetter DH, Schultz R, Percy AK, Glaze DG: A de novo X;3 translocation in Rett syndrome. Am J Med Genet 1990; 35: $148-151$.

25 Hagberg B, Goutieres F, Hanefeld F, Rett A, Wilson J: Rett syndrome: criteria for inclusion and exclusion. Brain Dev 1985; 7: $372-373$

25 Willard HF, Smith KD, Sutherland J: Isolation and characterization of a major tandem repeat family from the human $\mathrm{X}$ chromosome. Nucl Acid Res 1983; 11: 2017 -2033.

27 Wolfe J, Darling SM, Erickson RP et al: Isolation and characterization of an alphoid centromeric repeat family from the human Y chromosome. J Mol Biol 1985; 182: 477 - 485.

28 Rosenberg C, Janson M, Nordeskjold M, Borresen AL, ViannaMorgante AM: Intragenic reorganization of RB1 in a complex $(4 ; 13)$ rearrangement demonstrated by FISH. Cytogenet Cell Genet 1994; 65: $268-271$.

29 Rosenberg C, Navajas L, Vagenas DF et al: Clinical diagnosis of heterozygous dystrophin gene deletions by fluorescence in situ hybridization. Neuromusc Dis 1998; 8: 447-452.

30 de Pauw ES, Verwoerd NP, Duinkerken N et al: Assessment of telomere length in hematopoietic interphase cells using in situ hybridization and digital fluorescence microscopy. Cyt 1998; 32: $163-169$

31 Albertsen HM, Abderrahim H, Cann HM, Dausset J, Le Paslier D, Cohen D: Construction and characterization of a yeast artificial chromosome library containing seven haploid human genome equivalents. Proc Natl Acad Sci USA 1990; 87: 4256-4260.

32 Brownstein $\mathrm{BH}$, Silverman GA, Little RD et al: Isolation of singlecopy human genes from a library of yeast artificial chromosome clones. Science 1989; 244: $1348-1351$.

33 Brown CJ, Ballabio A, Rupert JL et al: A gene from the region of the human $\mathrm{X}$ inactivation centre is expressed exclusively from the inactive X chromosome. Nat 1991; 349: 38-44.

34 Dirks RW, Van de Rijke FM, Fujishita S, van der Ploeg M, Raap AK: Methodologies for specific intron and exon RNA localization in cultured cells by haptenized and fluorochromized probes. J Cell Sci 1993; 104: 1187 - 1197.

35 Allen RC, Zoghbi HY, Mosely AB, Rosenblatt HM, Belmont JW: Methylation of HpaII and HhaI sites near the polymorpuic CAG repeat in the human androgen-receptor gene correlates with $\mathrm{X}$ chromosome inactivation. Am J Hum Genet 1992; 51: 1229_ 1239.

36 Clemson CM, McNeil JA, Willard HF, Lawrence JB: XIST RNA paints the inactive $\mathrm{X}$ chromosome at interphase: evidence for a novel RNA involved in nuclear/chromosome structure. J Cell Biol 1996; 132: $259-275$.

37 Ellison KA, Roth EJ, McCabe ER, Chinault AC, Zoghbi HY: Isolation of a yeast artificial chromosome contig spanning the $\mathrm{X}$ chromosomal translocation breakpoint in a patient with Rett syndrome. Am J Med Genet 1993; 47: 1124-1134.

38 Migeon BR, Luo S, Jani M, Jeppesen P: The severe phenotype of females with tiny ring $X$ chromosomes is associated with inability of these chromosomes to undergo $\mathrm{X}$ inactivation. $\mathrm{Am}$ I Hum Genet 1994; 55: 497 - 504

39 Guillen DR, Lowichik A, Schneider NR, Cohen DS, Garcia S, Zinn AR: Prune-belly syndrome and other anomalies in a stillborn fetus with a ring X chromosome lacking XIST. Am J Med Genet 1997; 70: $32-36$. 